\title{
The Effects and Consequences of Blood Pressure Cuff Pulse Wave Filtering
}

\author{
Jiri Jilek \\ Carditech \\ Culver City \\ California \\ USA \\ jjilek30@gmail.com
}

\author{
Milan Stork \\ Department of Electronics and Information \\ Technology/RICE, University of West Bohemia, \\ University of West Bohemia \\ Plzen, Czech Republic \\ stork@kae.zcu.cz
}

\begin{abstract}
Cuff pulse waves together with cuff pressure are essential in modern automatic blood pressure (BP) devices. Most methods for BP determination employ cuff wave amplitudes. Physiological features of cuff waves have been neglected. In this study we described the origin of amplitude based approach to automatic BP determination. The cuff waves are commonly called oscillations and the methods for BP determination are called oscillometric methods. Excessive filtering of cuff waves causes obliteration of important physiological features of the waves. We introduced wider frequency band filtering that opens avenues to more sophisticated processing of cuff waves. Examples of cuff pulse wave applications beyond BP determination are shown.
\end{abstract}

Keywords - blood pressure; oscillations; cuff-pulse; pulse waves; pulse wave filters; systolic pressure; diastolic pressure; central pressure; hemodynamic variables

\section{INTRODUCTION}

Blood pressure (BP) measurement is frequently performed test in hospitals, clinics, and homes. Automatic BP monitors with just one cuff are the most prevalent BP devices on the market today. Most BP monitors use arm cuffs but wrist cuff devices are also popular. Single cuff devices usually use the cuff pressure and arterial pulses acquired from the cuff during BP tests. The pressure data from the cuff have the form of descending or ascending cuff pressure ramp which is modulated by arterial pulsations.

Arterial pulses and their contours were well known in 19th century. They were acquired by mechanical devices called sphygmographs and recorded usually by Ludwig recorder. An example of recorded radial artery pulse waves is in Fig 1. The recordings were accurate enough to show the basic physiological features of an arterial pulse. Different types of pulses were recorded by French physiologist Marey who performed experiments with counterpressure in which the hand was placed in a waterfilled chamber and a variable counter-pressure was

Milan Stork's participation was supported by Department of Applied Electronics and Telecommunications, University of West Bohemia, Plzen, Czech Republic and by the Ministry of Education, Youth and Sports of the Czech Republic under the RICE - New Technologies and Concepts for Smart Industrial Systems, project No. LO1607 and the Internal Grant Agency of University of West Bohemia in Plzen, the project SGS-2021-005 applied [1]. When the counter pressure was increased or decreased, the amplitudes of pulses in the chamber decreased. Due to large amount of water in the chamber and the submerged hand, substantial damping of pulse wave contours took place. Marey recognized the different nature of these pulses and called them oscillations. A recording of Marey's counter pressure experiment is in Fig 2. Comparison of pulse contours in Fig. 1 and 2 reveals the differences. The only useful features of pulse contours in Fig 2 are the amplitudes.

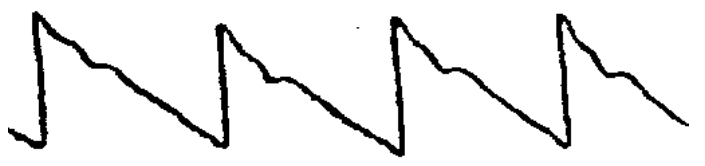

Figure 1. Radial arterial pulse waves obtained with a sphygmograph

Marey's concepts of counter-pressure and oscillations are important because 100 years later they became the foundation of BP oscillometry

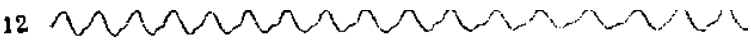

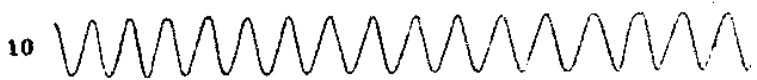
8

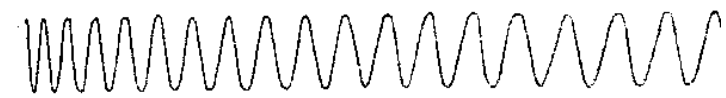

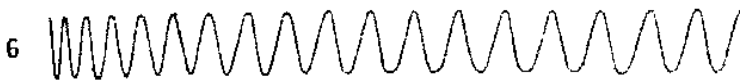
- WMNMNNANANA

Figure 2. Marey's counter-pressure experiment. The middle trace shows the maximum oscillation amplitudes

Arterial pulse waves elicited in the cuff and their changing amplitudes with changing cuff pressure became the basis of cuff-based automatic BP measurements. Posey and Geddes showed [2] that the maximum amplitude of pulses in the cuff corresponded to mean arterial pressure (MAP). When pressure in the cuff was increased above MAP or decreased below MAP, the amplitudes decreased. Posey and Geddes chose to use Marey's term oscillations and concentrated on their amplitudes. 
Geddes concept of oscillometric method [3] is shown in Fig. 3. The top trace shows the descending cuff pressure (CP) ramp and gradually emerging pulses superimposed on the CP ramp. This representation of the superimposed pulses is incorrect because the amplitudes keep increasing until the end of cuff deflation. The middle trace, called cuff pressure oscillations, contradicts the pulses in the upper trace. The middle trace oscillation amplitudes increase until they reach the maximum and then the amplitudes decrease until the end of the gradual cuff deflation. The middle trace shows wave shapes resembling Marey's oscillations in Fig. 2.

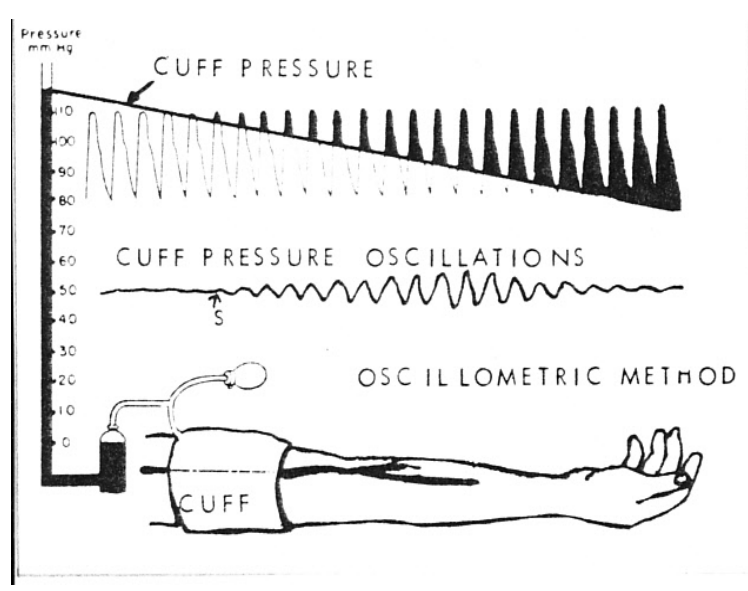

Figure 3. Geddes concept of oscillometric method

Geddes did not publish description of wave filters he and his colleagues used, but the waves (oscillations) in Fig 3 suggest narrow band filtering.

Many methods based on evaluation of cuff-pulse amplitudes have been described in literature. These pulses were commonly called oscillations in spite of the fact that they were not the same as pulse contours recorded by Marey (Fig 2). Published studies of oscillometric methods apparently used excessive filtering of cuff-pulse waves that obliterated details of the wave shapes. Very little information on cuff-pulse wave filtering has been published.

Details of cuff-pulse contours were mostly ignored until 2010 when Jilek disputed correctness of the term oscillations in the cuff [4]. Jilek's research showed that the cuff-pulses obtained at $\mathrm{CP}$ below DBP are arterial pulses that are similar to pulses obtained by other methods. Jilek introduced new term cuff-arterial pulse (cuff- pulse) instead of incorrect term oscillation. More detailed studies of cuff-arterial pulses were subsequently published [5-6]. Further evidence against the oscillometric terminology emerged. The methods and results in our paper concentrate on cuff pulse wave filtering that minimizes distortion and reveals fully formed arterial waves.

\section{METHODS}

The cuff arterial pulses have low amplitudes, typically 1-4 mmHg and are usually separated from the $\mathrm{CP}$ ramp and amplified before they are further processed. Fig. 4 shows descending cuff pressure ramp and separated cuff-pulse waves (CPW). The time-compressed waveforms do not reveal wave details.

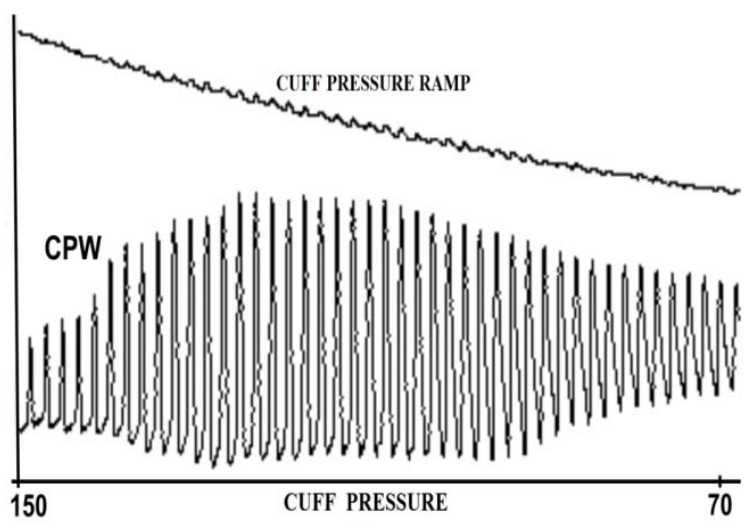

Figure 4. Separation of cuff pulse waves (CPW) from cuff pressure ramp

Block diagram of cuff-pulse transducing, amplification, and filtering used in our study is in Fig. 5. Pressure in the cuff is converted to voltage by pressure transducer and the output signal is amplified in the instrumentation amplifier. The low pass filter is a second order active filter with the cutoff frequency $f_{c}=35 \mathrm{~Hz}$. The output of the low pass filter is connected to the CP input of the ADC and to the input of the high pass filter. The high pass filter is a second order active filter with cutoff frequency $f c=0.5 \mathrm{~Hz}$. The $0.5 \mathrm{~Hz}$ cutoff frequency is sufficient for the removal of the $\mathrm{CP}$ descending ramp and for preservation of the low frequency components of cuff pulse waveforms. Higher ramp cutoff frequencies can result in the $\mathrm{CP}$ waveform distortion. The output signals of the filters are digitized and further processing is performed by software routines.

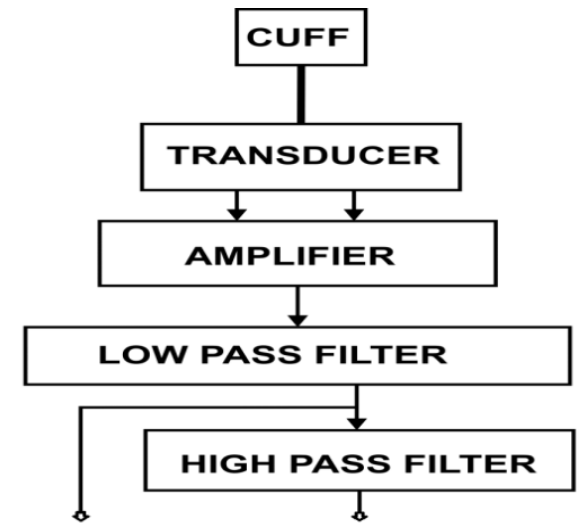

Figure 5.. Block diagram of cuff pressure and cuff pulse transducing and filters.

\section{RESULTS}

The filters described above (low pass $\mathrm{fc}=35 \mathrm{~Hz}$ and high pass $\mathrm{fc}=0.5 \mathrm{~Hz}$ ) produce cuff pulse waves that are under certain condition similar to arterial waves obtained by other methods (direct method, arterial tonometry). Cuff-pulse waves in Fig. 6 elucidate the changes they undergo during a gradual 
cuff deflation. Fig. 6a shows cuff waves $(\mathrm{CW})$ that are distorted from the start of the procedure to the point of DBP. The distortion is produced by turbulent blood flow and arterial wall vibrations under the cuff. Below the point of DBP the flow is laminar and the waves are no longer distorted. The transition from distorted to undistorted waves can be observed in Fig. 6b. Fig. 6c shows waves obtained at CP just below DBP. The waves are similar to waves obtained by invasive and noninvasive methods. Arterial waves obtained with the direct method are shown in Fig. 7.

Besides visual evaluation of cuff waves, a study from 2017 [7] compared cuff-pulse wave left ventricular ejection time (LVET) numerically to predicted LVET values developed by Weissler [8]. At mean heart rate $66 \mathrm{bpm}$, the mean LVET from wrist waves was 309 milliseconds and the predicted mean value was 304 milliseconds.

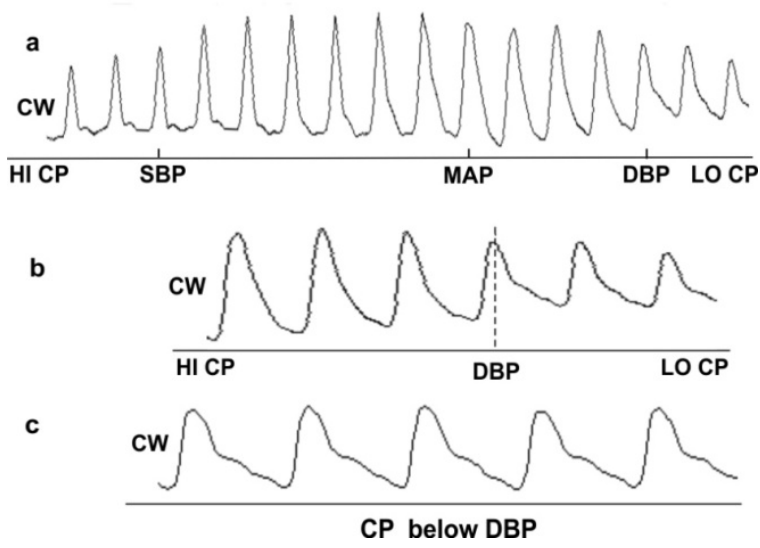

Figure 6 Cuff-pulse waves $(\mathrm{CW})$ acquired from a 62 years-old male. Fig. 6-a:cuff-waves $(\mathrm{CW})$ during a gradual $\mathrm{CP}$ deflation. Fig. 6-b: transition from distorted to undistorted waveforms. Fig. 6-c: CWs acquired at CP just below DBP

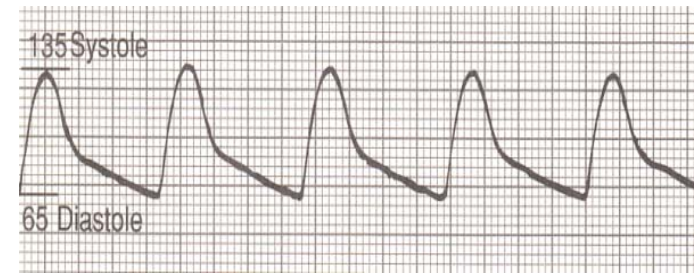

Figure 7. Pulse waves obtained with direct method

\section{Discussion}

Our studies of cuff-pulse waves obtained with wide-band filtering revealed 2 new visual and numerical features. First, cuff pulse waves are not oscillations as claimed by Geddes and other investigators [5-6]. Second, cuff-waves obtained at cuff pressures below DBP level are similar to waves obtained by other methods (Fig 7) and they can be used in applications that go beyond BP determination.

The concept of oscillations developed by Marey (Fig 2) was applied by Geddes and others to pulses in the cuff. Investigators of cuff pulses in the second part of the 20th century apparently assumed that cuff pulses were similar to Marey's oscillations (Fig. 2) and they used narrow band filtering and concentrated on cuff-pulse amplitudes. The waves in Fig. 3 are more similar to Marey's oscillations in Fig 2 than to the waves in Fig 6.

Amplitudes of pulses in the cuff (oscillations) continue to dominate investigation of oscillometric methodology. Geddes introduced the characteristic ratio method for determination of SBP and DBP values [9]. The method was based on a statistical estimate, not on physiology. According to Geddes, SBP corresponded to amplitude of about $50 \%$ of maximal amplitude and for DBP the ratio was about $80 \%$. The concept of amplitude envelope was developed and various methods have been published. A change of slope of the amplitude envelope method found supporters. A report describing the function of an oscillometric device [10] claims that the point of SBP corresponds to the point of initial increase in the pulse amplitude. Another report put SBP on the minimum ascending slope of the amplitude envelope and DBP on the maximal descending slope.

Different methods resulted in different BP values. There are many descriptions of oscillometric methods in scientific and engineering journals. Some authors show cuff waves (oscillations) but they are usually time-compressed and their shapes are not discernible [11]. Automatic electronic sphygmomanometers using oscillometric methods started appearing on the commercial market in the seventies of the 20th century. They were called oscillometric BP monitors. Microprocessors facilitated algorithmic methods for BP determination. Many commercial BP monitors using algorithmic methods have been developed. The manufacturers' algorithmic methods are considered intellectual property and are kept secret. The secrecy became a problem because experts interested in the methodology could only speculate about the nature of the commercial algorithms.

During the past decade cuff pulse waves attracted more attention. The concept of cuff pulse acquisition at cuff pressure below the point of DBP has been introduced [12].

Cuff-pulse waves acquired at CP below DBP have been used for estimation of some hemodynamic variables. Several commercial instruments for indirect estimation of central aortic pressure use brachial-cuff waves acquired at CP below the point of DBP [13-14]. Brachial cuff waves acquired with Sphygmocor Excel instrument are shown in Fig. 8

Estimation of stroke volume, cardiac output, total peripheral resistance, and systemic arterial compliance was accomplished with a dual-cuff experimental system [15]. The system used cuff-pulse waves obtained at CP just below the DBP level.

Cuff-pulse methods have the advantage of being operator independent and they can be fully automatic. Only application of the cuff is required. The disadvantage is the damping of higher frequencies caused by the air in the cuff and the soft tissue under the cuff. Better results are obtained with wrist cuff methods. Simultaneously acquired wrist cuff and arm cuff waves are shown in Fig. 9 Wrist cuff waves show more details because there is much smaller volume of air in the cuff and less soft tissue under the cuff. 


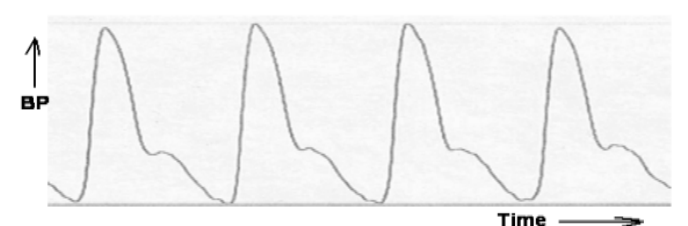

Figure 8. Sphygmocor Excel brachial waves obtained at CP below DBP

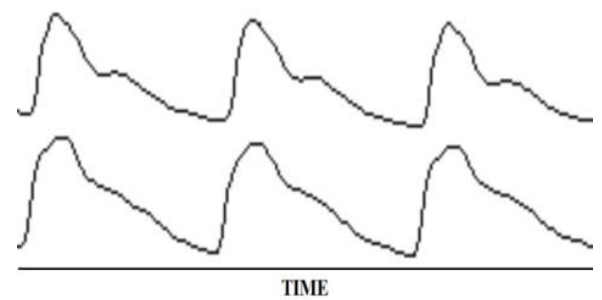

Figure 9. Wrist cuff (radial) and arm cuff (brachial) waves acquired simultaneously

Noninvasive arterial tonometry provides more accurate waves but it requires skilled application of the probe to the artery under investigation. The simplicity of cuff-pulse methods appears to be more acceptable in clinical environment.

\section{CONCLUSION}

Cuff- pulse waves obtained at CP below DBP with wide band filtering offers new applications for 21 st century. Functions of noninvasive BP monitors can be expanded to include additional hemodynamic variables that will help physicians to better diagnose and manage hypertension.

\section{REFERENCES}

[1] Marey, E.J. La Circulation du sang a l'etat physiologique et dans les maladies 1881, Paris, Masson.

[2] Posey, J.A. \& Geddes, L.A. The measuring of the point of maximum oscillations in cuff pressure in the indirect measurement of blood pressure. Cardiovasc Res Bul 1969, Vol. 8, pp 15-25
[3] Geddes LA. Handbook of Blood Pressure Measurement. Humana Press 1991.

[4] Jilek J. Oscillations or Cuff-arterial Pulses ? Biomedical Instrumentation \& Technology 2010;44:6.

[5] Jilek J, Stork M. Arterial Pulsations in the Blood Pressure Cuff: Are They Hemodynamic Pulses or Oscillations? International Journal of Biology and Biomedical Engineering 2012; 6(1):35-42.

[6] Jilek J., Stork M. The Contours of Arterial Pulsations in the Blood Pressure Cuff Are Hemodynamic Waveforms Rather Than Oscillations. WSEAS, Prague, CR, September 26-28, 2011: 88-90.

[7] Jilek J, Stork M. Assesing left ventricular ejection time from wrist cuff arterial waveforms. AE2017 International Conference Proceedings 2017, Plzen, Czech Republic.

[8] Weissler AM, Harris WS,Schoenfeld CD, Systolic time intervals in heart failure in man, Circulation 1968;21:149155.

[9] Geddes, L.A. Characterization of the oscillometric method for measuring indirect blood pressure. Ann Biomed Eng 1982, Vol. 10, pp 271-280.

[10] Borow KM, Newburger JW. Noninvasive estimation of central aortic pressure using the oscillometric method for analyzing systemic artery pulsatile blood flow; comparative study of indirect systolic, diastolic and mean brachial artery pressure with simultaneous direct ascending aortic pressure measurement. Am Heart J. 1982;103:879.

[11] Ringrose J, Sridar S. The elegance of simplicity. J Clin Hypertens 2020;22:1173-1176

[12] Stork M, Jilek J. Cuff Pressure Pulse Waveforms: Their Current and Prospective Applications in Biomedical Instrumentation. In: Laskovski A.N. ed. Biomedical Engineering, Trends in Electronics, Communications and Software; pp 193-210. Intech, January 2011.

[13] Peng X, Schultz MG, Picone DS, Dwyer N, Black JA, Roberts-Thomson P, Sharman JE. Non-invasive measurement of reservoir pressure parameters from brachialcuff blood pressure waveforms. J Clin Hypertens 2018;20:1703-1711.

[14] Pucci G, Cheriyan J, Hubsch A, Dickson SS. Evaluation of Vicorder, a novel cuff-based device for noninvasive estimation of central blood pressure. J Htn 2013;31:77-85.

[15] Jilek J, Stork M. Estimation of hemodynamics from arm blood pressure cuff waveforms. Int Conf Proc AE2013, Plzen, Czech Republic.. 